\title{
DISEÑO, DESARROLLO Y RESULTADOS DE UN PROGRAMA DE EDUCACIÓN SOCIO-EMOCIONAL PARA LA FORMACIÓN DE DOCENTES A NIVEL DE GRADO Y POSTGRADO
}

\author{
Raquel Palomera \\ Elena Briones \\ Alicia Gómez-Linares \\ Universidad de Cantabria
}

RESUMEN: En las últimas décadas la investigación educativa ha aumentado su interés sobre las emociones, tanto en relación a la salud del docente como por su implicación en su desempeño y eficacia profesional. Al tiempo, cada vez hay mayor evidencia del rol de las habilidades emocionales en el bienestar y desarrollo infanto-juvenil. Así, podemos encontrar una gran variedad de programas educativos dirigidos a la educación emocional en los escolares; sin embargo, apenas existen iniciativas para la formación docente, menos aún para su educación formal. En este trabajo presentamos dos propuestas pioneras dirigidas a la educación emocional, tanto en la formación inicial como permanente del docente. Una vez transcurridos varios años de implementación, y a través de varias evaluaciones, hemos recogido información valiosa que nos permite obtener conclusiones sobre su efectividad, así como identificar las metodologías más apropiadas para llegar a esos resultados. Precisamente por ello, parece necesario expandir esta formación a otros centros de educación superior, tanto por sus beneficios sobre el propio docente como por las potenciales consecuencias positivas en el desarrollo integral de sus estudiantes. Por tanto, la finalidad de este trabajo es inspirar y guiar futuras iniciativas dirigidas a la formación emocional y social de los docentes.

PALABAS CLAVE: Competencias socio-emocionales, docente, educación emocional, educación superior, evaluación.

\section{DESIGN, IMPLEMENTATION AND RESULTS OF A SOCIAL AND EMOTIONAL LEARNING PROGRAM FOR TEACHER TRAINING AT UNDERGRADUATE AND POSTGRADUATE LEVEL}

\footnotetext{
ABSTRACT: In the last decades, educational research has increased its interest on affects, not just in its relation to teacher's health but in its relation
} 
to his/her implication to the professional performance and efficiency. At the same time, there is an increasing evidence on the role of the emotional and social skills regarding welfare and development of the children and youngsters. In this way, there are a great variety of educational programs that address the emotional education of school children. However, there are just a few initiatives on teacher training, and less on formal training. In this article, two pioneer proposals, that address the emotional education for pre-service and professional teachers, are presented. Several years after its implementation, and evaluation, important information on the most appropriate methodologies to reach these results has been gathered. It seems necessary to expand this training to other higher education institutions, because of the benefits for teachers and the potential positive consequences on the student's development. So, the aim of this work is to inspire and guide future initiatives addressed to the emotional training of teachers.

KEYWORDS: Socio-emotional competences, teacher, emotional education, higher education, evaluation.

Recibido: 18/03/2016

Aceptado: 19/07/2016

Correspondencia: Elena Briones, Universidad de Cantabria, Facultad de Educación, Departamento de Educación, Avd. Los Castros s/n, 39005 Santander, Cantabria (España). Email: brionese@unican.es.

\section{INTRODUCCIÓN}

Históricamente, los docentes han tendido a prestar poca atención o ignorar el rol de las emociones en los procesos de aprendizaje. Desde Descartes ha existido un dualismo entre razón y emoción que ha permanecido hasta finales del siglo XX, cuando los estudios cognitivos y neuro-científicos han mostrado la estrecha relación entre ambos en su funcionamiento diario. Las emociones, lejos de interferir en la razón, son fundamentales para interpretar el mundo y manejarnos de forma adaptada en él. Este proceso de esclarecimiento científico ha ayudado a que actualmente los docentes sean conscientes de que enseñar implica tener en cuenta sus propias emociones y las de los demás.

Las emociones juegan un poderoso papel a la hora de determinar a qué prestamos atención y qué seleccionamos para recordar, también regulan nuestro comportamiento y guían nuestra asimilación del mundo que nos rodea (Medina, 2008). Por ello, las emociones asocian nuestro aprendizaje con placer o dolor. Si el estudiante experimenta emociones positivas, su cerebro amplifica su capacidad de aprendizaje (Fredrikson, 2001), mientras que el estrés la bloquea (Sylwester, 2007). A pesar de que los docentes no tienen el control absoluto del clima emocional del aula, están al tiempo ejerciendo una fuerte influencia sobre el mismo. A menudo, su comportamiento (consciente o inconsciente) verbal y no verbal, junto 
a su expresión emocional y actitudes tienen un gran efecto sobre sus estudiantes, favoreciendo o inhibiendo su aprendizaje (Powell y Kusuma-Powell, 2010).

El presente trabajo se basa en dos premisas. La primera es que los docentes que tienen muy desarrolladas sus habilidades emocionales crean entornos educativos más positivos, y favorecen de forma más efectiva el aprendizaje y en particular modelan el desarrollo emocional (ver revisión en Palomera, Fernández-Berrocal y Brackett, 2008); además, perciben una mayor satisfacción con su trabajo y presentan mejores índices de bienestar y salud, junto a una menor incidencia de burnout (Brackett, Palomera, Mojsa, Reyes y Salovey, 2010).

La segunda premisa es que las habilidades socio-emocionales se pueden desarrollar, como lo demuestra el relevante estudio de meta-análisis Ilevado a cabo por Durlak, Weissberg, Dymnicki, Taylor y Schellinger (2011), a partir de las experiencias de educación emocional en entornos escolares. Igualmente, un estudio conducido en el contexto de formación inicial del docente (en formato de seminario) mostró el incremento significativo de las competencias emocionales de los estudiantes en formación para ser docentes, y un mayor ajuste en la transición a la vida laboral (Byron, 2001).

Cuando hablamos de educación socio-emocional estamos haciendo referencia al proceso educativo que promueve el desarrollo de cinco competencias sociales y emocionales interrelacionadas (CASEL, 2005):

- Conocimiento de sí mismo: evaluar con precisión los sentimientos, intereses, valores y fortalezas de uno mismo; mantener un sentido bien fundamentado de confianza en sí mismo.

- Autogestión emocional: la regulación de las emociones de uno mismo para manejar el estrés, control de la impulsividad, y perseverar en la superación de obstáculos; establecer y monitorear el logro de metas personales y académicas; expresar emociones de manera apropiada.

- Conciencia social: ser capaz de ponerse en el lugar de los demás y empatizar, reconocer y apreciar las similitudes y diferencias individuales y de grupo; reconocer y utilizar los recursos de la familia, la escuela y la comunidad.

- Habilidades de relación: establecer y mantener relaciones sanas y positivas basadas en la cooperación; resistir la presión social inapropiada; prevenir, gestionar y resolver conflictos interpersonales; buscar ayuda cuando sea necesario.

- Toma de decisiones responsables: tomar decisiones basadas en el examen de las normas éticas, las preocupaciones de seguridad, las normas sociales apropiadas, respeto a los demás, y consecuencias probables de diferentes acciones; la aplicación de las habilidades de toma de decisiones a situaciones académicas y sociales; contribuir al bienestar de la escuela y la comunidad.

El meta-análisis de Durlak et al. (2011), al que hemos hecho referencia previamente, también mostraba los criterios de eficacia y fracaso de los programas de educación socio-emocional implementados en el entorno escolar. Los programas efectivos, es decir, aquellos que conseguían sus objetivos (relativos al desarrollo emocional y social), reunían cuatro características: empleo de una serie de sesiones 
cuidadosamente secuenciadas para desarrollar habilidades de un modo gradual; uso de formas activas de aprendizaje, lo cual implica que los alumnos practiquen sus nuevas habilidades y dicha ejecución sea evaluada; dedicación de tiempo y atención focalizada y adecuada para la formación de cada habilidad; la enseñanza explícita de habilidades concretas como objetivo, de manera que los alumnos sepan qué se espera de ellos.

Al contrario, los programas que no eran efectivos mostraban las siguientes dificultades: falta de fidelidad al ejecutar el programa; un elevado índice de rotación del profesorado; quejas de algunos profesores que alegaban que sus sustitutos tenían una formación menos adecuada y estaban menos preparados para la intervención; problemas en la coordinación y la gestión de la intervención por parte del centro.

Por otro lado, Kornaki y Caruso (2007), quienes implementan programas para el desarrollo emocional en el entorno empresarial, establecen una serie de orientaciones para la educación emocional en población adulta, tales como: insertar dinámicas de cohesión grupal; utilizar metodologías vivenciales y de experimentación, donde se utilizan los recursos y experiencias personales de los asistentes; trabajar con métodos grupales y cooperativos; realizar feedback y evaluaciones que informen de resultados; o dejar tiempos para la reflexión.

Ante los beneficios que comportan la educación emocional así como los criterios de éxito aportados por experiencias previas, nos propusimos formar en habilidades emocionales tanto a los futuros docentes, como a los que ya están en activo. Para ello, se implementó una materia troncal en el currículo del Grado en Magisterio en Educación Primaria y en Educación Infantil, así como el Máster en Educación Emocional, Social y de la Creatividad, título propio ofertado a los estudiantes de Magisterio como doble título de Grado y a los profesionales titulados. En este trabajo presentamos ambas experiencias pioneras, a nivel nacional e internacional, junto a los resultados encontrados hasta el momento.

\section{Formación inicial del docente}

En el año 2010, con el inicio de los nuevos Grados y el Plan Bolonia se incorpora el lenguaje de las competencias en el Espacio Europeo de Educación Superior (EEES), con importantes implicaciones en la ordenación de las titulaciones e identificación de las competencias que cada egresado debe alcanzar para desempeñar con calidad su labor profesional. De esta forma se renovaba el sistema de educación incorporando aspectos como las actitudes, los valores y las habilidades, que permiten a una persona desenvolverse con eficacia y autonomía en los nuevos entornos, heterogéneos y cambiantes. Dichos aspectos suponen los saberes que integran el término competencia (Delors, 1996): el saber conocer, el saber hacer, el saber ser y el saber convivir.

Ciñéndonos a la educación formal de los futuros docentes y a su diseño en nuestra Universidad, señalamos que este cambio supuso que los Grados en Magisterio en Educación Primaria y en Educación Infantil contemplaran la formación de competencias básicas, transversales y genéricas; así como competencias específicas de cada grado. Las competencias básicas suponen los objetivos expuestos en los Reales Decretos que establecen la ordenación de las enseñanzas universitarias oficiales 
(RD1393/2007 y RD861/2010). Mientras que las competencias transversales parten del posicionamiento realizado por nuestra universidad a las indicaciones realizadas en las disposiciones mencionadas.

La asignatura de Formación en valores y competencias personales para docentes, supone un resultado del compromiso de nuestra universidad de incorporar en los planes de estudio las habilidades, competencias y valores considerados fundamentales para la formación integral de sus titulados. De esta manera, los planes de estudio de Magisterio integraron dentro de la formación básica, la educación de estas competencias personales e interpersonales.

Formación en valores y competencias personales para docentes

La asignatura de Formación en valores y competencias personales para docentes (FVCPD) pertenece al módulo de Formación Básica y tiene un carácter obligatorio dentro de los planes de estudios de los títulos de Grado en Magisterio en Educación Infantil y en Educación Primaria en nuestra Universidad.

La asignatura supone 6 ECTS y es impartida de forma presencial en el primer cuatrimestre del primer curso, a través del cuerpo docente adscrito al Área de Psicología Evolutiva y de la Educación.

\section{Objetivos}

En este apartado se presentan los objetivos, resultados de aprendizaje y contenidos de la asignatura de FVCPD en ambos grados en Magisterio (véase una descripción en detalle en Palomera, Gómez-Pérez, Briones y Gómez-Linares, 2016), tomados de la Memoria para la Solicitud de Verificación del Título de Grado en Magisterio en Educación Infantil y en Educación Primaria y de las Guías Docentes de la asignatura en ambas titulaciones (Curso académico 2015-2016).

En cuanto a los objetivos, se dirigen a: reflexionar sobre la forma de ser y estar propia y del otro; desarrollar estrategias para el propio desarrollo socio-emocional y ético; promover el bienestar docente y la convivencia en la comunidad educativa.

Los resultados esperados de aprendizaje, por su parte, se concretan en:

- Conocer los derechos humanos y los derechos de la infancia.

- Analizar procesos y proyectos para el desarrollo de dichos derechos.

- Mostrar respeto y tolerancia hacia las diferencias y hacia los demás en las relaciones.

- Ser capaz de comunicarse de forma activa y eficaz.

- Ser capaz de comunicarse de forma asertiva, mostrar empatía, negociar en grupo, realizar y recibir críticas de forma eficaz. Mostrar habilidades sociales en la comunicación interpersonal.

- Ser capaz de percibir, expresar, comprender y regular las emociones propias y de los demás.

- Conocer, diseñar y ejecutar actividades de desarrollo socio-emocional y moral en la etapa de Educación Infantil / Educación Primaria (según corresponda). 
Los contenidos de la asignatura se concentran en la educación de competencias socio-emocionales y en la formación en valores, en concreto se priorizan los siguientes:

- Formación en los valores universales relacionados con los derechos humanos y los derechos de la infancia.

- Desarrollo de competencias que permitan el desarrollo de dichos valores y derechos.

- Desarrollo de competencias personales: comunicativas, emocionales y sociales.

- Programas de desarrollo de competencias personales y valores en Educación Infantil/Educación Primaria.

\section{Metodología}

En este artículo presentamos nuestra propuesta de educación socio-emocional y su evidencia empírica en una primera fase de implementación. Esta consiste en una formación teórica-práctica, a través de una metodología experiencial y cooperativa de las competencias emocionales (autoestima, empatía, autorregulación), sociales (asertividad y gestión de grupos), de comunicación y también en creatividad y en valores (otros resultados sobre la formación específica en valores puede consultarse en Briones y Lara, 2016; Briones, Palomera y Gómez-Linares, 2014; Briones, Palomera y Gómez-Linares, 2015).

Dada la importancia del trabajo en equipo para el desarrollo de la asignatura, su evaluación y el propio entrenamiento de competencias socio-emocionales, cuyo éxito depende en gran medida del clima creado en clase, se llevan a cabo diversas actividades para garantizar la confianza y el compromiso con el aprendizaje. De esta manera, se realizan actividades tales como la descrita en la Tabla 1, que contribuyen a la constitución del grupo de expertos, a establecer metas referidas al proceso seguido para alcanzar los objetivos del grupo, y al establecimiento de compromisos, que sin duda facilitan el entendimiento entre los integrantes. Los acuerdos en esta materia entre los estudiantes, reciben el nombre de contrato de aprendizaje, y serán revisados a lo largo del curso, en sesiones dirigidas a identificar elementos clave del funcionamiento eficaz de un grupo. Esta segunda actividad, les ofrece la oportunidad de resolver los posibles conflictos que hubiesen surgido hasta el momento, y de optimizar su funcionamiento grupal y relaciones entre los integrantes del grupo.

Tabla 1. Ejemplo de actividad para la formación del grupo y establecimiento de compromisos

\section{CONTRATO DE APRENDIZAJE}

ACTIVIDAD EN GRUPO: El funcionamiento correcto de un grupo de trabajo donde todos cooperan y se comprometen con el éxito del grupo y con el aprendizaje de todos sus integrantes, es esencial en el desarrollo de la asignatura. Por ello, es necesario que todos los integrantes sean conscientes de qué actividades y comportamientos se espera de ellos, se comprometan a asumirlos y se esfuercen en mantener el compromiso.

COMPROMISO: Cada uno de los integrantes del grupo nos comprometemos a:

1. Dedicar al estudio y trabajo personal el tiempo suficiente para lograr los objetivos grupales. 
2. Dedicar tiempo y espacio para desarrollar las actividades no presenciales según lo acordado por el grupo.

3. Asistir a las reuniones de trabajo y respetar los horarios.

4. Mostrar respeto por los demás y el tratamiento de la información personal.

5. Prestar atención cuando los demás hablen o expongan una opinión.

6. Discutir y acordar, en cada sesión, el plan de trabajo a seguir y elegir un miembro que actúe como moderador y organizador.

7. Discutir conjuntamente las dificultades encontradas y el planteamiento de cada actividad propuesta.

8. Repartir el esfuerzo de manera equitativa y por consenso.

9. Participar activamente y con buena disposición en las actividades de grupo.

10. Aportar lo que esté en mi mano para la mejora del grupo.

En a de de 20

Firma de todos los miembros del grupo

\section{Programa}

El programa de la asignatura es progresivo al abordar las competencias señaladas, dedicando el tiempo y los procedimientos específicos para cada una de las competencias siguiendo las recomendaciones de CASEL (2005), además de una metodología activa y cooperativa para abordar los contenidos teóricos propios de las competencias entrenadas.

La secuencia seguida comienza con el desarrollo del primer bloque de la asignatura correspondiente a la formación en valores. En la edición que se detalla en este documento, se siguió una metodología que integraba exposiciones de los docentes; prácticas; así como la utilización de la estrategia cooperativa denominada técnica puzle. En la aplicación de la técnica puzle se siguieron los pasos básicos descritos por sus autores (Aronson y Patnoe, 2011). Así, se comenzó por dividir los contenidos en los siguientes cuatro segmentos, seleccionando lecturas académicas para cada uno de ellos:

- Educación y Derechos Humanos. Contenidos de una pedagogía de la convivencia.

- Derechos del menor. Vulnerabilidad, riesgo y maltrato.

- La escuela y el docente en la protección del menor. La detección y notificación de situaciones de desprotección infantil.

- La intervención educativa y la colaboración entre escuela y agentes sociales.

Después del estudio de cada lectura por los denominados grupos de expertos, desarrollando para ello las actividades propuestas, se constituyen los grupos de formación, integrados por representantes de estudiantes de cada temática. Con objeto de generar aprendizaje sobre todos los materiales, resuelven un caso que requiere del análisis de una situación contextualizada abordando las distintas temáticas de las lecturas trabajadas. Finalmente, cada grupo de formación expone al gran grupo el análisis y soluciones que han elaborado y propuesto para resolver el caso. 
El rol del profesor en el aula consiste en aclarar las dudas que se presentan en cada fase, así como en orientarles en su resolución, animándoles a que consulten el material suministrado y otras posibles fuentes bibliográficas.

Se continúa con la dedicación en un segundo bloque a la formación específica en competencias socio-emocionales clave para la función docente. Este bloque es abordado de forma experiencial a través de diversas metodologías. Cada semana se aborda una temática relacionada con alguna competencia personal o interpersonal docente. Las temáticas abordadas en este bloque y su programación son las siguientes:

- Marco conceptual: Inteligencia emocional.

- Comunicación eficaz: Comunicación verbal y no verbal.

- Procesos grupales: Trabajo eficaz en equipo.

- Procesos grupales: Liderazgo en equipos de trabajo.

- Regulación emocional interpersonal. Autoeficacia, satisfacción y burnout en los docentes.

- Autoestima y empatía en la educación.

- Asertividad y resolución de conflictos interpersonales.

- Creatividad e innovación en la educación.

- Educando para la felicidad.

Esta temáticas se distribuyen entre los grupos de expertos creados en el bloque de valores. En esta ocasión los grupos deben realizar una exposición en clase sobre los contenidos teóricos de la competencia que se les haya asignado, empleando para ello las lecturas propuestas. Además, planifican una actividad para entrenar dicha competencia en clase. Estas exposiciones son precedidas por una tutoría en la cual se revisa dicha exposición, asimismo se contempla una tutoría posterior para la visualización conjunta de la grabación de la exposición y feedback grupal.

Además de las exposiciones grupales y explicaciones del profesor todas las semanas se dedica un tiempo a practicar la competencia que corresponda realizando diversas actividades mediante role-playing, modelado, moldeado, dinámicas grupales, ejercicios individuales de introspección, relajación, etc. Un ejemplo de las actividades que se llevan a cabo es la que se refleja en la Tabla 2, destinada al entrenamiento de la competencia comunicativa.

Tabla 2. Ejemplo de una actividad para el entrenamiento de la comunicación verbal y paraverbal

\begin{tabular}{|l|l|}
\hline \multicolumn{2}{|c|}{ Profesores que dejan huella } \\
\hline Objetivos & $-\begin{array}{l}\text { Identificación y valoración de los elementos importantes de las habilidades } \\
\text { de comunicación interpersonal (en uno mismo y en otros). } \\
-\end{array}$ \\
& $\begin{array}{l}\text { Toma de consciencia de las implicaciones de las competencias socio- } \\
\text { emocionales del docente. }\end{array}$ \\
& $\begin{array}{l}\text { Conexión de los aprendizajes sobre competencias socio-emocionales y en } \\
\text { concreto, habilidades comunicativas, con las vivencias personales de los } \\
\text { estudiantes. }\end{array}$ \\
\hline
\end{tabular}




\begin{tabular}{|l|l|}
\hline $\begin{array}{l}\text { Indicaciones } \\
\text { para su } \\
\text { desarrollo }\end{array}$ & \begin{tabular}{l} 
1. Uno por uno de cada subgrupo narra cómo fue su mejor o peor profesor. \\
2. Mientras se van sucediendo las narraciones individuales, los demás del \\
subgrupo observan las competencias comunicativas del narrador/a, \\
identificando una fortaleza y una debilidad comunicativa. \\
3. Cada subgrupo consensúa la narración más llamativa y el/la mejor \\
comunicador/a. \\
4. Al azar, 2 ó 3 mejores comunicadores/as exponen las mejores historias. \\
$\begin{array}{l}\text { 5. Puede darse el caso de que las experiencias que cuenten los/as } \\
\text { comunicadores no sean las suyas, sino la de otros compañeros/as. }\end{array}$ \\
\hline Resultados
\end{tabular}$\quad \begin{array}{l}\text { En la reflexión que surge en el aula posterior a la realización de la actividad, } \\
\text { se evidenciará tanto las características propias de las competencias socio- } \\
\text { emocionales clave de los docentes, como lo relativo a las propias habilidades } \\
\text { comunicativas y su importancia para la actividad docente. }\end{array}$ \\
\hline
\end{tabular}

La evaluación de la asignatura es continua y participativa, así los estudiantes se involucran en la evaluación de las competencias propias y de los compañeros empleando para ello dos rúbricas. En una de ellas, se evalúa el proceso (véase en Tabla 3 ), es decir la actitud y el compromiso en las actividades de grupo, la propia (autoinforme) y de cada integrante (heteroinforme).

Tabla 3. Rúbrica para la autoevaluación y heteroevaluación de los estudiantes en los grupos de expertos

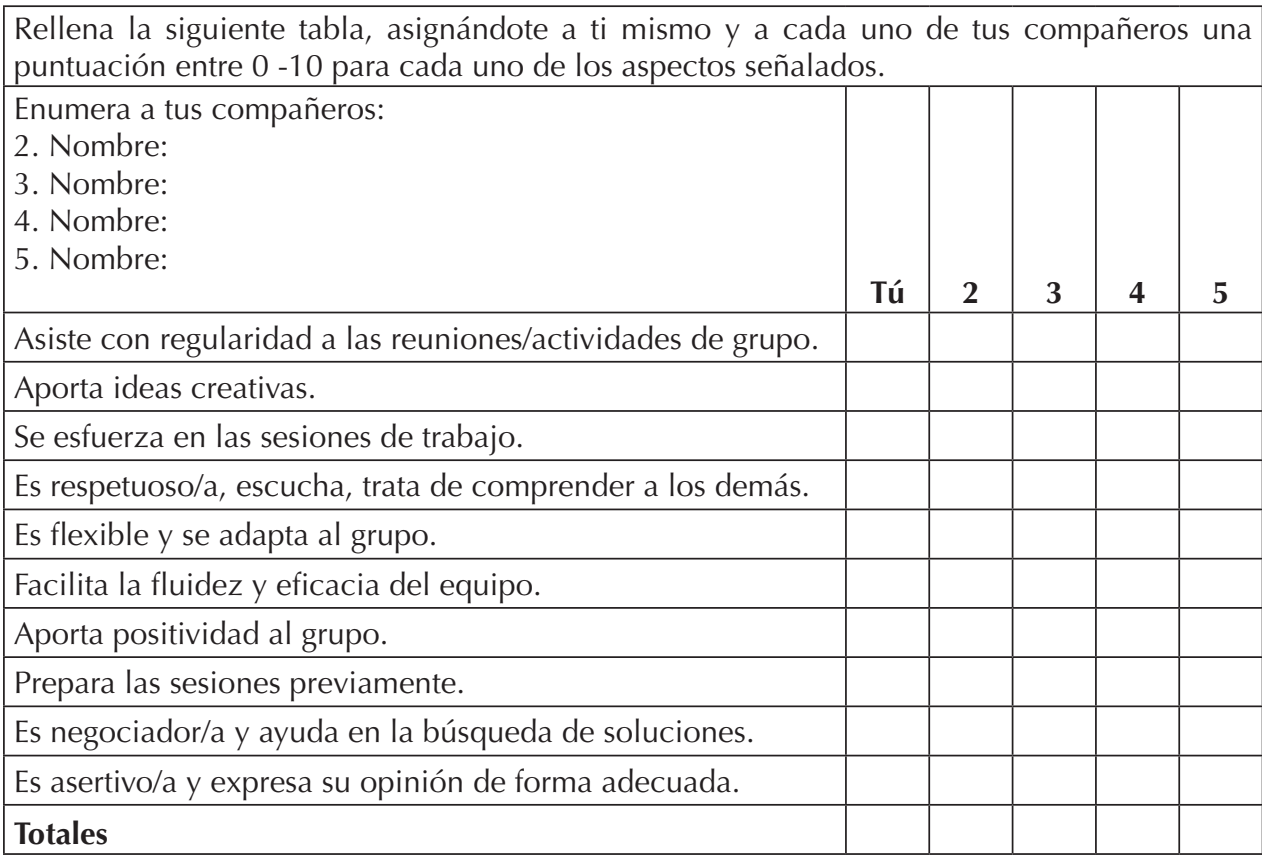

Además, para evaluar el producto, es decir, las exposiciones y trabajos de los grupos de expertos, se emplea la rúbrica descrita en la Tabla 4. Esta rúbrica es utilizada 
por el profesor y otros dos grupos de expertos que actúan de observadores-evaluadores en el aula. También, la evaluación de las competencias socio-emocionales cuenta con una prueba objetiva final sobre los contenidos teóricos.

Tabla 4. Rúbrica de evaluación de la exposición y trabajo del grupo de expertos

Señalad el grado de calidad de cada apartado para cada ponente o para el grupo, según vuestra opinión. Escribid una nota numérica final para cada ponente. Firmad por detrás los evaluadores presentes. También, podéis añadir algún comentario en el reverso.

\begin{tabular}{|c|c|c|c|c|c|c|c|c|c|c|c|c|c|c|c|}
\hline \multirow{3}{*}{$\begin{array}{c}\text { Criterios de Evaluación } \\
\text { Contenido }\end{array}$} & \multicolumn{15}{|c|}{ Integrantes del Grupo de Expertos evaluado } \\
\hline & \multicolumn{3}{|c|}{1} & \multicolumn{3}{|c|}{2} & \multicolumn{3}{|c|}{3} & \multicolumn{3}{|c|}{4} & \multicolumn{3}{|c|}{5} \\
\hline & $\frac{\widetilde{\sigma}}{\mathscr{\sigma}}$ & $\frac{\frac{\sigma}{\sigma}}{2}$ & $\frac{\pi}{2}$ & $\cdot \frac{\pi}{\pi}$ & $\frac{\frac{\pi}{\sigma}}{2}$ & $\frac{\pi}{\pi}$ & $\frac{\widetilde{\pi}}{\widetilde{\pi}}$ & $\frac{.0}{\frac{\pi}{8}}$ & $\stackrel{\frac{\pi}{E}}{\frac{\pi}{<}}$ & $\cdot \frac{\widetilde{\sigma}}{\tilde{ల}}$ & 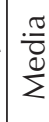 & $\stackrel{\Xi}{\frac{\pi}{<}}$ & $\frac{\widetilde{\sigma}}{\widetilde{\widetilde{\pi}}}$ & 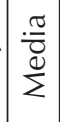 & $\frac{\pi}{<}$ \\
\hline \multicolumn{16}{|l|}{ Exhaustividad de contenidos. } \\
\hline \multicolumn{16}{|l|}{ Relaciona conceptos. } \\
\hline \multicolumn{16}{|l|}{$\begin{array}{l}\text { Uso apropiado de ejemplos, } \\
\text { imágenes, anécdotas }\end{array}$} \\
\hline \multicolumn{16}{|l|}{ Otro/s: } \\
\hline Formato & $\mathrm{B}$ & M & A & $\mathrm{B}$ & M & A & B & M & A & B & M & A & B & $M$ & A \\
\hline \multicolumn{16}{|l|}{ Explicación clara y ordenada } \\
\hline \multicolumn{16}{|l|}{$\begin{array}{l}\text { Uso adecuado de recursos } \\
\text { didácticos, } \mathrm{TIC}, \ldots\end{array}$} \\
\hline \multicolumn{16}{|l|}{$\begin{array}{l}\text { Lenguaje adaptado a la audiencia, } \\
\text { técnico }\end{array}$} \\
\hline \multicolumn{16}{|l|}{$\begin{array}{l}\text { Anima a participar con preguntas, } \\
\text { verifica el seguimiento }\end{array}$} \\
\hline \multicolumn{16}{|l|}{ Se ajusta a los tiempos } \\
\hline \multicolumn{16}{|l|}{$\begin{array}{l}\text { Uso de lenguaje no verbal } \\
\text { relajado, positivo, clarificador }\end{array}$} \\
\hline \multicolumn{16}{|l|}{ Otro/s: } \\
\hline Valoración general & $\mathrm{B}$ & $M$ & $\mathrm{~A}$ & $\mathrm{~B}$ & $M$ & A & B & $M$ & A & B & M & A & B & $M$ & A \\
\hline \multicolumn{16}{|l|}{ Dominio de la materia } \\
\hline \multicolumn{16}{|l|}{ Nivel de esfuerzo, implicación } \\
\hline \multicolumn{16}{|l|}{ Coordinación del grupo } \\
\hline \multicolumn{16}{|l|}{ Interés suscitado } \\
\hline \multicolumn{16}{|l|}{ Calidad de las respuestas } \\
\hline \multicolumn{16}{|l|}{$\begin{array}{l}\text { Creatividad, originalidad de la } \\
\text { presentación }\end{array}$} \\
\hline \multicolumn{16}{|l|}{ Otro/s: } \\
\hline Puntuación & & & & & & & & & & & & & & & \\
\hline
\end{tabular}


La evaluación del bloque de formación en valores se realiza sobre el trabajo grupal entregado por los estudiantes, relativo a la resolución de un caso, y tareas de preparación realizadas en el grupo de expertos.

\section{Formación permanente en postgrado}

En el curso académico 2011/2012 comenzó el Máster en Educación Emocional, Social y de la Creatividad, en el marco del Campus de Excelencia Internacional de la Universidad, mediante un convenio de colaboración y codirección entre la Fundación Botín y la Facultad de Educación. Se trata de un Título Propio Universitario único a nivel internacional que además ofrece la posibilidad de obtener una doble titulación de Grado y Máster al mismo tiempo. Actualmente se ha iniciado la V Edición.

\section{Máster en Educación Emocional, Social y de la Creatividad}

El Máster pretende favorecer el bienestar y desarrollo personal y social desde el contexto escolar, una de las necesidades más relevantes en la sociedad actual, debido fundamentalmente a los siguientes motivos:

- La infancia y la adolescencia son periodos de formación, madurez y desarrollo cruciales en nuestra vida, aunque también es cierta la capacidad de aprendizaje a lo largo de toda la vida, y por tanto la educación continua;

- La realidad social nos enfrenta a la necesidad de formar en habilidades para la vida;

- El sistema escolar es una pieza fundamental en la educación integral del alumnado;

- La formación del profesorado es un requisito imprescindible para poder lograr una educación de calidad, con efectividad y salud docente.

En este Máster se ofertan cada edición 41 plazas, de las cuales 15 están becadas por la Fundación Botín y 1 por la Universidad. El perfil de entrada es preferentemente de profesionales de la educación formal y no formal, aunque cada vez hay más demanda de otros perfiles profesionales. Los estudiantes solicitan este Máster a través de una presentación del CV en una entrevista personal y demostrando un nivel de B2 en inglés (pues parte de las clases se desarrollan con profesionales internacionales).

\section{Objetivos}

Al finalizar el Máster, el alumnado será capaz de:

- Utilizar su propia competencia emocional, social y creativa para alcanzar un mayor bienestar personal y social.

- Mejorar la eficacia de los procesos de enseñanza-aprendizaje mediante la utilización de estas competencias y las artes como un medio excepcional para ello.

- Dominar distintas herramientas de intervención psico-educativa a partir del conocimiento de programas innovadores de todo el mundo.

- Incorporar a su trabajo la promoción del bienestar y de las competencias emocionales, sociales y creativas durante la infancia y la adolescencia. 
- Crear, a partir de la discusión y la reflexión, las condiciones educativas necesarias para que esto ocurra con efectividad.

\section{Metodología}

La metodología empleada es teórico-práctica, con sesiones eminentemente prácticas y está basada en los criterios de éxito mencionados previamente. Se desarrolla a lo largo de varias sesiones semanales, con el apoyo de una plataforma virtual para el alojamiento de materiales y comunicación entre las diversas promociones. La mayoría del profesorado procede de otros centros nacionales e internacionales tanto de educación superior, como de centros educativos o profesionales de creatividad y de las artes. Todos ellos son referentes en su ámbito de estudio y trabajo.

La evaluación, llevada a cabo por la codirección y profesorado del Máster, se divide en módulos, siendo grupal para los tres primeros e individual para el practicum y Trabajo Fin de Master (TFM), y se realiza a través de pruebas a final de curso. Las pruebas grupales suponen una exposición de 15 minutos en las que tienen que responder, de la forma más creativa posible a diversas preguntas planteadas al inicio de curso en relación a lo aprendido y sus posibilidades de aplicación práctica. En el portafolio de practicum analizan el contexto en el que realizan las prácticas y plantean propuestas de mejora o planes de innovación en este campo. Finalmente, en el TFM defienden en un formato de Congreso de pósteres sus propuestas de innovación mediante evaluación por pares (donde siempre está presente el tutor del TFM). Los criterios de evaluación son: ajuste a los objetivos del trabajo; presentación formal/ académica, sintética, clara y organizada; fundamentación teórica; calidad de las propuestas (grado de innovación y creatividad); creatividad en la presentación.

\section{Programa}

Está diseñado como un postgrado de 60 créditos, dividido en cinco grandes módulos:

- Marco General y Competencias del docente (20 ECTS): En este módulo primero se presentan los fundamentos de la educación emocional, social y creativa; es decir, el qué, cómo, cuándo y por qué. En la segunda parte se trabaja de forma experiencial en las diferentes habilidades con el objeto de que los docentes desarrollen las mismas.

- Experiencias innovadoras en el ámbito educativo y modelos de buenas prácticas (20 ECTS): Diferentes científicos presentan los programas desarrollados a nivel nacional e internacional que destacan por su validez e innovación. A su vez, profesionales de la educación y las artes les inspiran como ejemplos de buenas prácticas a la hora de implementar innovaciones en este campo. De esta forma disponen de las dos miradas, la científica y la profesional.

- Educación para el bienestar (5 ECTS): Base teórica y práctica para fomentar la felicidad y bienestar en las escuelas y sus habitantes, como objetivo último de toda educación.

- Practicum (12 ECTS): los alumnos aplican durante su estancia en los centros escolares procesos de análisis y propuestas de mejora para promover un estilo 
de enseñanza basado en el estímulo y atención al desarrollo emocional, social y creativo de los educandos y el propio profesional. Otro perfil de estudiantes aplica programas de innovación también en centros de educación no formal, organizaciones, etc., con poblaciones diversas.

- Trabajo Fin de Máster (3 ECTS): diseño en formato póster de una propuesta de innovación educativa en este campo.

\section{Método}

A continuación presentamos las metodologías empleadas para investigar el impacto de las enseñanzas ofrecidas desde la formación inicial y de postgrado.

\section{Formación inicial: Formación en valores y competencias personales para docentes}

En un estudio reciente (Palomera, Briones, Gómez-Linares y Vera, 2016) exploramos el impacto del programa de formación $(\mathrm{N}=250)$ Ilevando a cabo una investigación cuasi-experimental con medida pretest y postest, y grupo control (de primer curso de otras Facultades de Educación, 25\% de la muestra). Los estudiantes respondieron en dos ocasiones (pre y post la formación) de forma anónima a una serie de escalas clásicas en este campo de estudio orientadas a evaluar algunas de las competencias entrenadas (empatía, autoestima, afectos, confianza para hablar en público y asertividad); también se evaluó la deseabilidad social y los cinco grandes rasgos de personalidad, como medidas de control.

Mediante un estudio cualitativo (Gómez-Linares, Palomera y Briones, 2014) empleando una encuesta semi-estructurada, anónima y por escrito, comprobamos la valoración de la experiencia educativa por parte de los estudiantes $(\mathrm{N}=263)$. El análisis de contenido se realiza sobre la información aportada a cuatro preguntas abiertas (su aprendizaje, lo que les ha gustado más y menos, y sugerencias para la mejora de la asignatura).

\section{Formación de postgrado: Máster en Educación Emocional, Social y de la Creatividad}

Cada año, los estudiantes (hasta el momento $\mathrm{N}=115$ ) rellenan de forma anónima una escala de satisfacción con las sesiones desarrolladas por cada profesor una vez que éstas han finalizado. Esta escala de satisfacción emplea una escala Likert de 5 puntos ( $1=$ Nada Satisfecho; $5=$ Muy Satisfecho) para evaluar los siguientes criterios: los contenidos presentados han resultado comprensibles e interesantes; la metodología empleada es adecuada a los objetivos propuestos; la comunicación docente ha sido accesible; los recursos educativos aportados han resultado de ayuda; grado de satisfacción con los ponentes, el aprendizaje obtenido y con la organización. Asimismo, los estudiantes puntúan en porcentaje el grado de comprensión lingüística cuando el ponente es de habla inglesa. Finalmente, disponen de un apartado de observaciones para comentar lo que consideren.

Por otro lado, al final de cada edición ( $\mathrm{N}=63)$, los estudiantes rellenan de forma anónima un cuestionario online acerca de su grado de satisfacción. Con la información así obtenida se realiza un análisis de contenido. 


\section{Resultados}

A continuación presentamos los resultados encontrados hasta el momento en relación al impacto de las enseñanzas ofrecidas desde la formación inicial y de postgrado.

\section{Formación inicial: Formación en valores y competencias personales para docentes}

Los resultados del último estudio realizado (Palomera et al., 2016) demostraron que el programa había tenido un impacto significativo en la autoestima, la empatía, la confianza como orador (sobre todo en relación con el miedo a hablar en público) y el afecto negativo de los estudiantes. Con respecto a la empatía, el programa promueve la fantasía y la preocupación empática, aunque parece ser necesario dedicar más tiempo y un tratamiento específico para cambiar habilidades cognitivas complejas, tales como la toma de perspectiva. Mientras que la falta de cambio en la asertividad, parece indicar la necesidad de entrenar esta competencia incidiendo en que los estudios de caso y el role playing partan de las vivencias de los estudiantes.

Por otra parte, el análisis de la información obtenida mediante el estudio cualitativo (Gómez-Linares et al., 2014) nos permite documentar que el alumnado percibe haber aprendido valores y competencias para la vida personal y profesional (categoría AVC, p. ej. conocer y valorarse a uno mismo y a los demás, afrontamiento positivo y autorregulación, habilidades sociales y resolución de dilemas, a ser más feliz). En los siguientes testimonios podemos detectar dichos aspectos:

"He aprendido a conocer los valores que me forman y a trabajar una serie de estrategias para comprender con mayor facilidad a los niños, apoyarlos. También me han servido para mi vida diaria, actuar de diferente manera en ciertas situaciones" (AVC.12).

"He aprendido nuevas habilidades sociales para enfrentarme a la vida. Además, he aprendido a empatizar más y a ser más asertiva. Observar qué vía de actuación adopto en diferentes actuaciones fomentando cada vez más la asertividad" (AVC.55).

Por otra parte, detectamos que a los estudiantes les había gustado la metodología participativa, dialógica y experiencial. En concreto, realizaron comentarios positivos relativos a la exposición y prácticas realizadas en grupos; la relajación; y el clima de libertad creativa, confianza y diversión creada en el aula (categoría POS).

"Me ha gustado mucho trabajar en el grupo de expertos, nos hemos sabido escuchar y hemos sacado nuestro trabajo obteniendo buenos resultados" (POS.32).

No obstante, también se identificaron algunos aspectos menos satisfactorios, por ejemplo la forma de trabajar los contenidos teóricos o la evaluación de una actividad concreta (categoría NEG):

"Que no dependan las explicaciones tanto de los alumnos" (NEG.247).

"Tweets [ejercicio semanal de síntesis del tema]...valorados solo por el docente" (NEG.101). 
Finalmente, los estudiantes expresaron algunas sugerencias referidas a cambios metodológicos y de evaluación concretos (categoría SUG), como por ejemplo:

"Lecturas menos complejas y más breves" (SUG.3).

\section{Formación de postgrado: Máster en Educación Emocional, Social y de la Creatividad}

A lo largo de los cinco años de implementación, los docentes participantes han recibido puntuaciones medias comprendidas entre 4 y 5 puntos, lo que indica un alto grado de satisfacción, que también se ve reflejado en sus observaciones. El grado medio de comprensión de las clases en inglés según los propios estudiantes ha sido del $80 \%$ de las sesiones. El feedback que nos aportan en cuestiones referidas a la metodología, comunicación o interés por ciertos contenidos, es trasladado a los ponentes para que sean tenidas en cuenta y de este modo puedan ajustar cada año las sesiones.

Del análisis cualitativo de la información sobre la satisfacción de los estudiantes presentamos a continuación ejemplos de sus comentarios relativos a diversos criterios:

- La metodología: "Cada ponente ha utilizado una metodología diferente con nosotros, eso nos ha dado dinamismo y a mí personalmente me ha gustado [...]. No se trata de clases magistrales donde el ponente transmite sus contenidos, sino que en todo momento son participativas, en las que lo que pensamos o sentimos ha sido importante" (MET.6).

- Los contenidos del programa: "En lo referido a los contenidos estoy encantada, es más, considero que son necesarios y que muchos de ellos se deberían dar en la carrera a todos los alumnos por su gran utilidad" (CON.100).

- El aula: "Mucho mejor aula que el año pasado, permite una comunicación e interacción mayor tanto con tus compañeros como con el aula. En lo que al espacio respecta, la hemos movido y adecuado a nuestras necesidades siempre que lo hemos necesitado" (REC/ES.25).

- Los recursos: "El moodle, es un recurso que se agradece ya que te permite usar documentos online y, además, usarla como una especie de "agenda electrónica" que te mantiene al tanto de las diferentes actualizaciones. Así como compartir recursos con otros compañeros, dudas, preguntas..." (REC/EDU.25).

- Los ponentes: "Los docentes han sido unos expertos en el tema, además de simpáticos, nos han motivado a seguir con el cambio que nos hemos propuesto en la educación [...]. Han demostrado la actitud y la aptitud necesaria para transmitirnos lo que estaba fijado, y en muchos casos, mucho más" (DOC.89).

- El idioma: "No me ha resultado difícil seguir las clases en inglés, lo único que si me ha merecido de una concentración extra para poder así enterarme de todo, por lo que debido a la cantidad de horas que pasamos en la universidad, había días que se me hacía muy cansado, sobre todo mentalmente";"Pienso que ojalá hubiera más clases en inglés ya que ayuda a aprender en inglés ciertos conceptos que ya sabemos en español" (LING.113).

- La organización: "La organización me ha parecido la correcta, puesto que en todo momento se ha contado con nosotros sobre todo para tomar las de- 
cisiones que más nos afectan. Creo que es excelente, ya que el trabajo que requiere me parece muy difícil. Siempre tenemos todos los materiales en moodle antes de las clases, cualquier cambio es notificado con antelación, y siempre pendientes de nosotros como personas, ya no sólo como alumnos" (ORG.1).

- Comentario libre: "En general mi satisfacción con el máster es de sobresaliente, ya que me ha ofrecido y proporcionado mucho más de lo que me esperaba de él, se lo recomiendo a todo docente que quiera tener una visión diferente y creativa de dar clase y de trabajar en el aula" (SAT/GL.40).

\section{Conclusiones}

Partiendo de nuestra experiencia pionera y validada en educación emocional y social durante seis años, hemos descrito dos propuestas que deseamos sirvan como modelo de buenas prácticas para inspirar el diseño de la formación en esta materia en otros espacios de la educación superior.

La conclusión más importante que podemos extraer de los estudios desarrollados en el Grado en Educación es que es posible mejorar las competencias socio-emocionales en la formación inicial de los maestros. Lo cual nos estimula a seguir desarrollando el modelo educativo introduciendo algunas mejoras con objeto de generar un mayor impacto en competencias socio-emocionales tales como la asertividad y la empatía, así como estudiar empíricamente el efecto en otras en las que no se ha probado su eficacia: creatividad, toma de decisiones y gestión de grupo.

Por otro lado, los estudiantes del Máster nos devuelven un feedback muy positivo, que nos anima a seguir con el esfuerzo emprendido y también nos ha permitido ajustar cuestiones importantes en la secuenciación de contenidos, métodos de agrupación y evaluación, o los horarios para facilitar el seguimiento. En donde más diversidad de opiniones se han detectado ha sido en relación a la dificultad para seguir las clases en inglés, que obviamente depende del nivel de partida de los estudiantes; en general, lo explican en relación al esfuerzo cognitivo extra que supone, aunque también depende del acento de procedencia del profesor; por otra parte, señalan que les resulta más complicada la expresión que la comprensión en el idioma inglés, aunque se ayudan entre ellos en este punto cuando es necesario.

A través de la triangulación de los resultados derivados de la experiencia de grado y postgrado concluimos que los estudiantes se sienten satisfechos con su formación en relación a los contenidos ofertados, con la metodología empleada y con la utilidad percibida para aplicar el aprendizaje adquirido en su vida cotidiana y académica. Por otro lado, al menos en el grado podemos confirmar que han desarrollado sus competencias socio-emocionales.

Mediante este documento pretendemos asimismo, incidir en la necesidad de rigurosidad a la hora de implementar este tipo de formación, y el interés común de realizar evaluaciones de los programas elaborados contando con la valoración de todos los participantes. También, compartimos la idea de que dedicarse a la educación implica un trabajo de investigación-acción constante, que conlleva la inmersión en distintas metodologías de enseñanza-aprendizaje. En este sentido, hemos comenza- 
do a abordar la formación en valores a través de las múltiples opciones que ofrece la argumentación dialógica como complemento imprescindible para la aplicación constructiva de las nuevas competencias adquiridas.

Igualmente, estamos explorando la articulación del aprendizaje-servicio en diversas modalidades que la educación superior ofrece para la formación socio-emocional.

Finalmente, hacemos notar que los proyectos innovadores requieren del compromiso, la formación continua y la coordinación del profesorado, así como de la flexibilidad de las instituciones para acoger dichas innovaciones.

\section{REFERENCIAS BIBLIOGRÁFICAS}

Aronson, E. y Patnoe, S. (2011). Cooperation in the classroom: The jigsaw method (3 ed.). Londres: Pinter y Martin.

Brackett, M. A., Palomera, R., Mojsa-Kaja, J., Reyes, M. R. y Slovey, P. (2010). Emotion-regulation ability, burnout, and job satisfaction among British secondaryschool teachers. Psychology in the Schools, 47(4), 406-417.

Briones, E. y Lara, L. (2016). Educación ética en la Universidad a través del diálogo multicultural online. Comunicar, 47(24), 99-107.

Briones, E., Palomera, R. y Gomez-Linares, A. (2015). Teaching in values in higher education: innovation by online dialogue between students from different universities. En International Conference on Education and New Developments, END 2015, (pp. 194-198), Porto (Portugal).

Briones, E., Palomera, R. y Gómez-Linares, A. (2014). Dilemas de la formación en valores en los grados de Magisterio. En XI Foro Internacional de la Evaluación de la Calidad de la Investigación y de la Educación Superior. Bilbao (España).

Byron, C. M. (2001). The effects of emotional knowledge education in the training of novice teachers. Digital Dissertation, 62(05).

Collaborative for Academic, Social, and Emotional Learning. (2005). Safe and sound: An educational leader's guide to evidence-based social and emotional learning programs-Illinois edition. Chicago, IL: Collaborative for Academic, Social, and Emotional Learning. Recuperado de: http://goo.gl/D5aTrz.

Delors, J. (1996). La educación encierra un tesoro. Informe a la UNESCO de la Comisión internacional sobre la educación para el siglo XXI. Madrid: Santillana/ UNESCO. Recuperado de: http://www.unesco.org/education/pdf/DELORS_S.PDF.

Durlak, J. A., Weissberg, R. P., Dymnicki, A. B., Taylor, R. D. y Schellinger, K. B. (2011). The impact of enhancing students' social and emotional learning: A metaanalysis of school-based universal interventions. Child development, 82(1), 405432. DOI: http://doi.org/10.1111/j.1467-8624.2010.01564.x.

Fredrickson, B. L. (2001). The role of positive emotions in positive psychology: the broaden-and-build theory of positive emotions. American Psychology, 56, 218226. Recuperado de: http://www.ncbi.nlm.nih.gov/pmc/articles/PMC3122271/.

Gómez-Linares, A., Palomera, R. y Briones, E. (2014). Formación en valores y competencias personales para docentes. Valoración cualitativa del alumnado. En el XI Foro Internacional de la Evaluación de la Calidad de la Investigación y de la Educación Superior. Bilbao (España). 
Kornacki, S. A. y Caruso, D. R. (2007). A theory-based, practical approach to emotional intelligence training: ten ways to increase emotional skills. En J. Ciarrochi y J. Mayer (eds.), Applying Emotional Intelligence: A Practitioner's Guide (pp. 53-88). NuevaYork: Psychology Press.

Medina, J. (2008). Brain rules: 12 principles for surviving and thriving at work, home, and school. Seattle, WA: Pear Press.

Palomera, R., Fernández-Berrocal, P. y Brackett, M. A. (2008). Emotional intelligence as a basic competence on teacher's initial education. Electronic Journal of Research on Educational Psychology, 6(2), 437-454.

Palomera, R., Gómez-Pérez, E., Briones, E. y Gómez-Linares, A. (2016). Formación en valores y competencias personales para docentes. En E. Briones y A. GómezLinares (coords), Psicología para docentes. Guía y casos resueltos aplicando el Aprendizaje Basado en Problemas (ABP) (pp. 45-52). Madrid: Pirámide.

Palomera, R., Briones, E., Gómez-Linares, A. y Vera, J. (2016). Filling the gap: improving the social and emotional competencies of pre-service teachers. Journal of Psychodidactics, 22(2).

Powell, W. y Kusuma-Powell, O. (2010). Becoming an emotionally intelligent teacher. Thousand Oaks, CA: Corwin.

Real Decreto 1393/2007, de 29 de octubre, por el que se establece la ordenación de las enseñanzas universitarias oficiales (BOE 30/10/2007). Recuperado de: https:// www.boe.es/buscar/act.php?id=BOE-A-2007-18770.

Real Decreto 861/2010, de 2 de julio, por el que se modifica el Real Decreto 1393/2007, de 29 de octubre, por el que se establece la ordenación de las enseñanzas universitarias oficiales (BOE 03/07/2010). Recuperado de: https://www. boe.es/buscar/doc.php?id=BOE-A-2010-10542.

Sylwester, R. (2007). The adolescent brain: researching for autonomy. Thousand Oaks, CA: Corwin. 